\title{
Vamos papear sobre dataviz? Uma experiência de conversas sobre visualização de dados durante a pandemia
}

\author{
Shall we talk about dataviz? An experience of conversation about data visualization \\ during pandemic times
}

Luiz Torres Ludwig, Joy Helena Worms Till, Flavia Nizia da Fonseca Ribeiro

visualização de dados, ensino não formal, áudio, pandemia

\begin{abstract}
Muitas experiências emergiram da situação imposta pela pandemia vivida mundialmente nos últimos dois anos. Este artigo traz o relato de uma prática proposta após a observação e constatação de algumas condições que alunos e professores passaram a viver em seu cotidiano, acadêmico ou não, tais como tempo excessivo diante das telas, os inúmeros recursos tecnológicos com os quais tiveram que lidar, dentre outros. Pinçando deste cotidiano a vontade de propor espaços informais de discussão de temas e de evitar as horas de exposição mediante a tela, nasce o PapoDado: sessões semanais de discussão e reflexão acerca do tema da visualização de dados, tendo como ambiente redes sociais de áudio, como numa conversa telefônica coletiva. Ainda que recente, esta vivência vem nos mostrando que, ainda que sem nenhum suporte visual, a discussão sobre a visualização de dados se mostra cada dia mais fértil, seja na diversidade de áreas de interesse que vem atraindo, ou no desdobramento dos assuntos propostos a cada uma das sessões. Este texto buscou trazer as primeiras análises dos impactos e conteúdos derivados dos encontros já propostos e, também, apontar os próximos passos para esta pesquisa ora em andamento.
\end{abstract}

data visualization, non-formal learning, audio, pandemics

Many experiences have emerged from the situation imposed by the global pandemic that the world has been experiencing for the past two years. This paper presents an account of one of these proposed practices, after observing and examining some conditions that students and teachers came to live in their daily lives, academic or not, such as an excessive screen time, a countless technological resources with which they had to deal with etc. From our pandemic daily life came the idea to propose an informal novideo required space for discussing topics, usually limited to academic dynamics. PapoDado is created as a weekly session for discussion and reflection on data visualization held on audio-only social networks, as if it was a collective phone conversation. Although recent, this experience has shown us that, even without any visual support, due to the nature of the platform where PapoDado takes place, the discussion on data visualization is proving to be every day more fertile, bringing a diversity of areas of interest to the discussion and unfolding different subjects every session. This text sought to bring the first considerations of the impacts from the meetings already held and also, to point out the next steps for this ongoing research.

Anais do $10^{\circ} \mathrm{CIDI}$ e $10^{\circ} \mathrm{CONGIC}$

Kelli C.A.S. Smythe, Rafael de Castro Andrade (orgs.)

Sociedade Brasileira de Design da Informação - SBDI

Curitiba | Brasil | 2021
Proceedings of the $10^{\text {th }} \mathrm{CIDI}$ and $10^{\text {th }}$ CONGIC

Kelli C.A.S. Smythe, Rafael de Castro Andrade (orgs.)

Sociedade Brasileira de Design da Informação - SBDI Curitiba | Brazil | 2021 


\section{Introdução}

Durante esse longo período em que estamos confinados, distantes fisicamente do trabalho, de amigos e família, muitas foram as ferramentas de reuniões on-line incorporadas ao nosso cotidiano. Num primeiro momento, as videochamadas foram extremamente bem-vindas, nos aproximando, através da interface digital e conexão em tempo real. No entanto, a intensidade de uso dessas, tanto para relações sociais como para atividades laborais, trouxe uma saturação aos usuários, em especial em relação à imagem na tela. (Ramachandran, 2021)

Dentre as atividades laborais atingidas pela nova realidade imposta pela pandemia, este artigo parte da experiência dos autores em suas práticas docentes e de como outros educadores têm enfrentado situações em que os alunos não se mostram confortáveis em permanecer com a câmera ligada, algo que parece ter se tornado cada vez mais comum. Muitas questões podem influenciar este comportamento, tais como desconforto em mostrar seu ambiente familiar, falta de privacidade, excesso de estímulos visuais, entre outras. De todo modo, ter a imagem observada pelos colegas e professores e também ver nossa própria imagem durante as chamadas de vídeo claramente traz um grande cansaço.

Nesse contexto, as redes sociais baseadas em áudio, como ClubHouse e Twitter Spaces, eliminam a necessidade de estarmos sozinhos em um ambiente ou adequadamente vestidos. Um fone de ouvido e um acesso simples por aplicativo nos permite participar de conversas mais informalmente, como numa chamada telefônica coletiva.

Éramos dois professores universitários ávidos por construir um espaço de troca mais descontraído e democrático e também, cientes dos desafios como propor a inserção das pessoas em mais um ambiente digital, ainda que com características diferentes, e um ainda maior que seria abordar um assunto de natureza majoritariamente visual somente pelas vozes dos envolvidos. Tendo como premissa criar um espaço descontraído, aberto à contribuições, onde pudéssemos discutir sobre visualização de dados, design de informação e temas correlatos, iniciamos nossa sala de debates em áudio.

Desafio acolhido e colocado em prática, até o momento da escrita deste texto, foram 13 (treze) eventos semanais organizados ao longo de mais de 3 meses. Este trabalho tem como objetivo relatar a experiência da estruturação das sessões de bate-papos sobre o tema da visualização de dados via áudio, nas plataformas ClubHouse e Twitter Spaces e apresentar algumas conclusões sobre o processo e as discussões realizadas.

\section{Visualização de dados via áudio}

Com a crescente produção e consumo de dados pela sociedade e a consequente necessidade de compreendê-los e analisá-los, ao longo dos anos surgiram eventos, encontros e grupos que promovem a discussão sobre o tema. Como uma das áreas de estudo relacionada a dados, a visualização de dados emerge como um assunto profícuo situado na interseção entre o design e dados. Também conhecida como visualização de informação - ou simplesmente infovis ou dataviz, a visualização de dados é definida pelo autor Andy Kirk (2012) como "a representação e 
apresentação de dados que exploram nossas habilidades de percepção visual para amplificar a cognição". A partir dessa definição, percebemos que a visualização de dados exerce um papel importante na mediação entre os dados e o público. Em uma sociedade cada vez mais orientada por dados, não só o acesso aos dados é importante mas também acesso às ferramentas adequadas para analisá-los. (Diamond, 2011 apud Kosminsky et al. (orgs.), 2018) Nesse contexto, a visualização de dados aparece como área relevante de estudo nos dias de hoje que traz benefícios quando utilizadas. Acerca das vantagens da utilização de visualizações de dados, segundo Diamond,

"visualizações permitem a comparação de um conjunto de valores; a ilustração dos relacionamentos entre pontos de dados; a indicação das partes de um sistema e o relacionamento e a interação dessas partes; a criação e as interpretações de mapas; o rastreamento de mudanças ao longo do tempo; e a análise de texto" (Diamond apud Kosminsky et al. (orgs.), 2011)

Com o maior interesse pela visualização de dados, vemos nascer eventos, encontros e grupos a esse respeito no Brasil. No contexto nacional, temos eventos periódicos como "DataViz.Rio" ${ }^{1}$, publicações on-line como "DataVizBr"², e podcasts que valorizam a visualização de dados como "Visualmente" ${ }^{3}$. Esses são alguns exemplos de iniciativas independentes que promovem o debate em torno dessa área no contexto brasileiro. Cada grupo possui sua própria dinâmica, plataforma de encontro, público e foco temático. No início de 2021, assistimos a explosão da rede social de áudio ClubHouse ${ }^{4}$, estimulando outros serviços a criarem plataformas semelhantes. Em março, foi lançada a funcionalidade Spaces no serviço Twitter ${ }^{5}$, com funções muito similares, porém ainda restrita a alguns usuários selecionados. Por alguns consideradas descendentes dos podcasts, as ferramentas sociais para conversas por áudio oferecem ambientes bastante informais e atraentes para aproximar interessados em temas específicos.

\footnotetext{
1 Iniciativa que organiza eventos presenciais e virtuais sobre visualização de dados. Disponível em <https://datavizrio.wixsite.com/datavizrio> acessado em 15 de maio de 2021.

2 Organização e produção de publicações on-line. Disponível em <https://medium.com/datavizbr> acessado em 15 de maio de 2021.

3 Podcast voltado para a discussão de design, artes e humanidades. Disponível em <https://visualmente.com.br/> acessado em 15 de maio de 2021.

${ }^{4}$ A rede ClubHouse foi lançada em abril de 2020, inicialmente apenas para usuários de iPhone, e disponível somente para convidados. Projetada para permitir conversas baseadas em áudio entre amigos e outras pessoas, em todo o mundo. Nos primeiros meses de 2021, foi um dos aplicativos mais baixados da AppStore, com 10 milhões de instalações somente no mês de fevereiro. Disponível em <https://exame.com/tecnologia/as-redes-sociais-e-a-apostano-audio-o-que-ficou-do-efeito-clubhouse> acessado em 15 de maio de 2021.

${ }^{5}$ O Twitter Spaces é lançado em março de 2021, em versão para Android, com uma proposta muito semelhante, numa alternativa para conversas em áudio. Sem a necessidade de convite, funciona dentro do aplicativo Twitter. Disponível em <https://blog.twitter.com/en_us/topics/product/2021/spaces-is-here.html> acessado em 15 de maio de 2021.
} 


\section{PapoDado}

Diante do cenário anteriormente exposto, nasce o PapoDado, com a intenção de promover a conversa informal sobre visualização de dados, em um ambiente que favorecesse a participação dos mais diversos interessados, dando voz a pesquisadores, professores, alunos e profissionais convidados. Como já comentado, num primeiro momento a rede social de áudio ClubHouse, com acesso via celular, nos pareceu adequada para investigar um novo tipo de construção de aprendizagem.

A proposta de salas em grupo, abertas para os participantes da rede, nas quais as conversas acontecem em tempo real e não podem ser gravadas, se assemelham à celebrações ou eventos festivos nos quais usufruímos daquele momento e, após a despedida, os bate-papos ali realizados permanecem apenas na memória de quem participou. Nas salas, as pessoas podem participar como ouvintes ou como oradores, somente por áudio, sem texto ou imagens. O responsável pela sala pode convidar quem estiver apenas ouvindo para falar e, no sentido inverso, quem está só ouvindo pode pedir a palavra. Isso permite muita fluidez nas falas, potencializando olhares diversos a partir da proposta de tema inicial.

Por meio de uma interface gráfica no celular, os participantes têm poucas possibilidades de interação, gerando uma curiosa subversão de funcionalidades. Por exemplo, clicar no botão do microfone repetidamente, na cultura da ferramenta, adquiriu o significado de aplausos ao locutor, durante sua fala. 
Figura 1: foto da interface da rede social ClubHouse

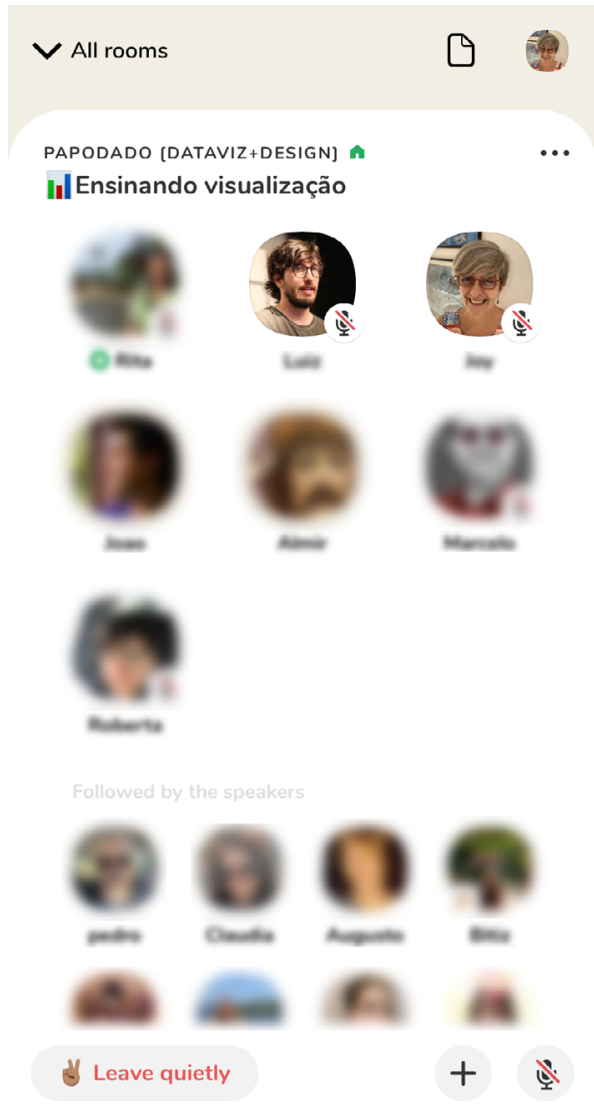

Durante dez edições, nos reunimos no ambiente do ClubHouse. Porém, a restrição de uso para apenas usuários de iPhone, produziu um grande desconforto em relação à acessibilidade e migramos para a ferramenta Twitter Spaces, que, como já mencionado, tem uma proposta bastante semelhante para as conversas em áudio.

A migração permitiu ampliarmos nossa lista de convidados, tanto para oradores que pudessem contribuir com suas expertises nos assuntos propostos, como para interessados em geral, usuários dos mais diversos celulares e sistemas operacionais. Se, por um lado, ampliamos a acessibilidade às conversas, por outro a ferramenta ainda apresenta algumas instabilidades, o que nos tem gerado alguma insegurança nos encontros. Ainda assim, continuamos explorando este espaço até o momento em que escrevemos este texto, tendo lá já realizado 3 encontros.

Dessa forma, em ambos os ambientes utilizados, estruturamos nossos encontros semanais em temas específicos que tivessem a potencialidade de se desdobrar dependendo do olhar de cada um dos participantes, convidando a cada edição colegas especialistas nos assuntos propostos. Educadores, estudantes, designers, programadores, advogados, entre outros profissionais atuantes em frentes diversas formam a mesa inicial de oradores de cada dia. Além disso, como as salas são abertas aos frequentadores da rede, surgem curiosos das mais diversas áreas que podem se manter como ouvintes ou solicitar a palavra para suas contribuições, gerando conversas efetivamente interdisciplinares. Cabe aos moderadores 
avaliarem se abrem ou não os microfones para os requisitantes, considerando o momento da discussão. O público que vem frequentando os encontros é diverso, porém compartilha o interesse na transformação que os dados promovem na sociedade. Profissionais e estudantes, cujas áreas de atuação têm sido afetadas pela presença cada vez mais relevante de dados computacionais, constituem a maior audiência. Desse modo, já estiveram no PapoDado, estudantes e profissionais das áreas de Engenharia, Ciência de Dados, Biomedicina, Educação, Design, Comunicação, Direito etc

Figura 2: foto da interface do espaço do PapoDado na rede social Twitter Spaces.

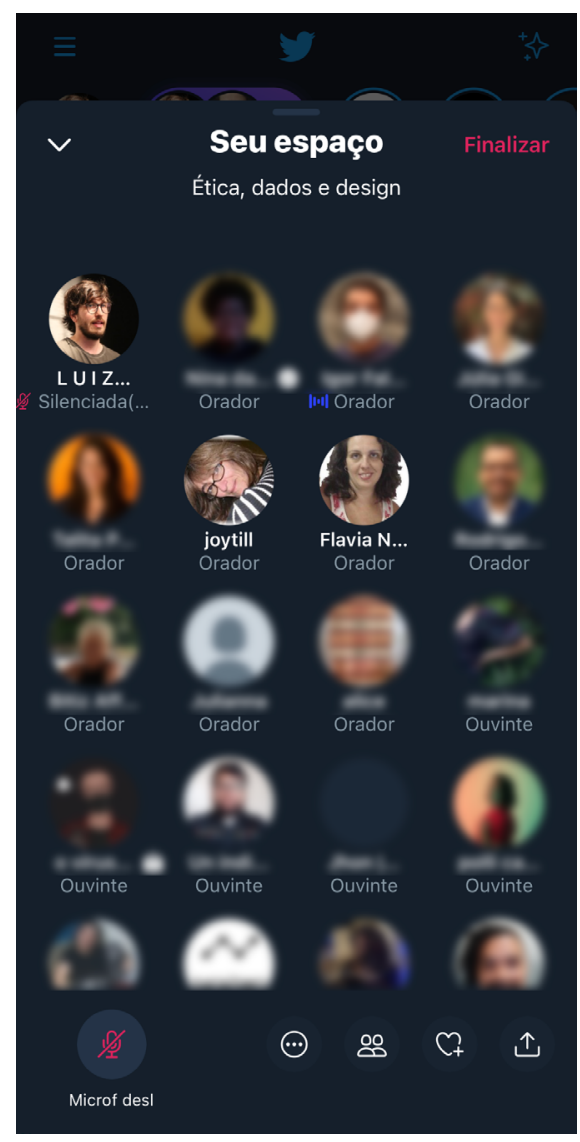

O ambiente descontraído se mostrou interessante pois gerou uma grande espontaneidade nos debates, numa mistura de contribuições dos convidados para a mesa inicial com as dos participantes que pediam a palavra. O formato de conversa permite a ocorrência de desvios naturais que fazemos ao reunir pensamentos diversos, sem a preocupação de chegar a conclusões definitivas. Com esta natureza, os temas de cada reunião surgem com base nos eventos que as precederam. Como definimos a duração de cada encontro para aproximadamente uma hora, os assuntos não se esgotam, mas sim, introduzem novos caminhos que seguem para as reuniões seguintes. 


\section{Como um tema levou ao outro}

A cada edição, os organizadores apresentaram temas para que funcionassem como disparadores em cada conversa. Como mencionamos, à medida que os participantes iam sendo incorporados como "locutores", novas questões surgiam, levando a caminhos não previstos e produzindo uma construção compartilhada do pensamento, em um processo interativo de inteligência coletiva. (Lévy, 2003, p. 121)

Figura 3: linha do tempo: panorama geral dos temas propostos nas sessões contempladas na análise desse artigo.

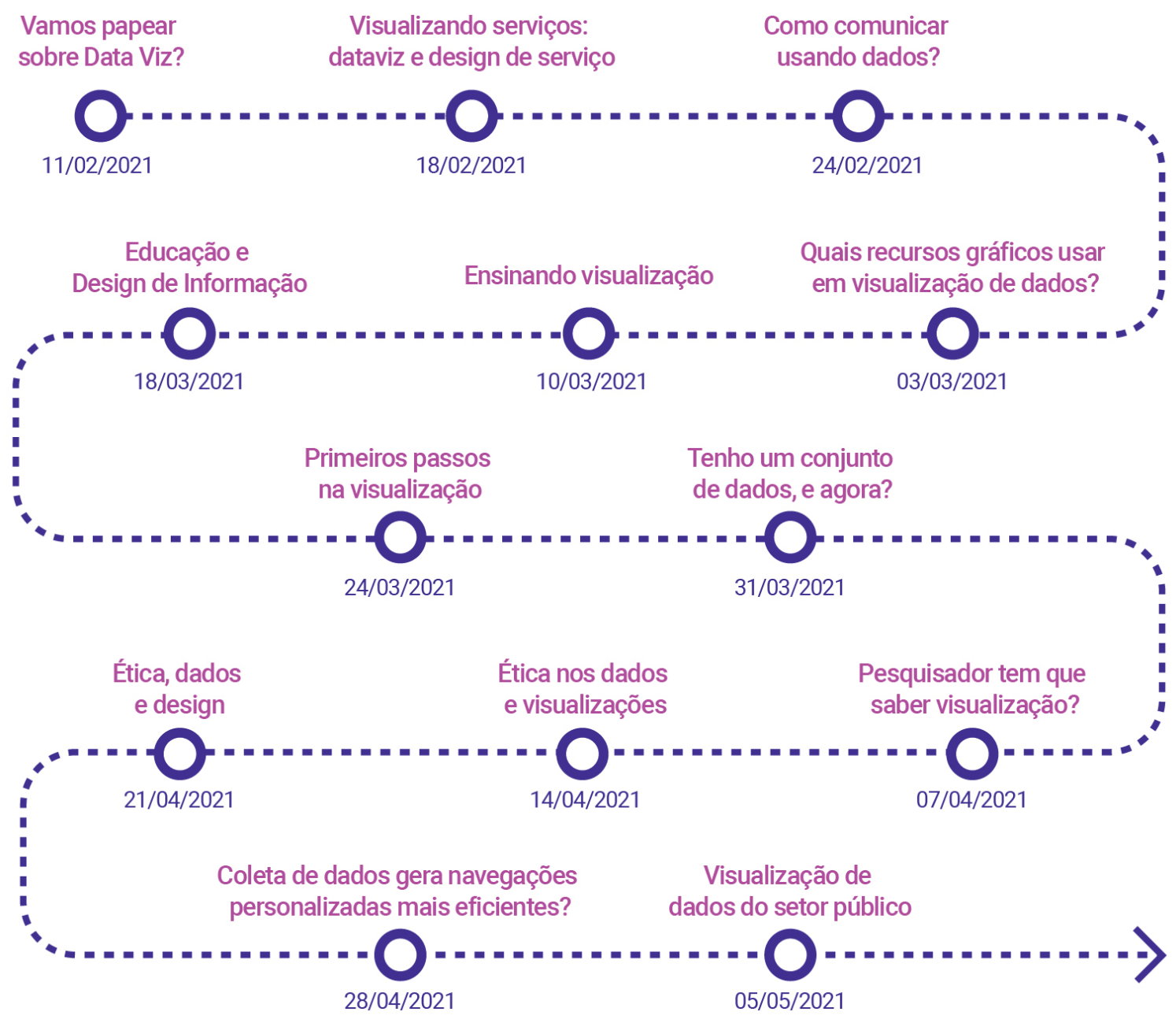

Nosso primeiro encontro realizou-se em 11/02/21 com a seguinte chamada: 'Vamos papear sobre DataViz?'. A proposta era um bate-papo sobre visualização de dados, ao mesmo tempo, em que experimentávamos juntos pela primeira vez a interface do ClubHouse como organizadores de uma sala. Até aquele momento, confinados há quase um ano, visualizações sobre a pandemia foram a tônica dos debates.

O segundo evento, em 18/02, teve como tema 'Visualizando serviços: dataviz e design de serviço'. Convidamos profissionais e pesquisadores que reafirmaram a importância da clareza 
do design de informação em projetos de design de serviços e também a relevância da criação de mapas para discutir e entender melhor os fluxos dos serviços.

O encontro de 24/02, intitulado 'Como comunicar usando dados?', nos proporcionou conversar sobre o trabalho desde o momento da coleta e estratégias de visualização. Refletimos sobre a necessidade de conhecimento de linguagens de programação ou da possibilidade de utilização de softwares comerciais para a construção de visualizações. Com 16 participantes, levantamos questionamentos ligados à literacia visual que nos levou ao tema da sessão seguinte.

'Quais recursos gráficos usar em visualização de dados?' foi como nomeamos nossa quarta conversa, em 03/03. Compreender quais recursos gráficos podem ser usados para comunicar dados objetivando seu melhor entendimento foi o ponto de partida da conversa partilhada por um grupo de 23 pessoas. Metáforas, indicadores visuais (visual cues), elementos gráficos suscitaram o interesse do grupo. Encaminhamos para o evento seguinte a discussão sobre o ensino de visualização.

No nosso quinto encontro, 'Ensinando visualização', em 10/03, participaram educadores de universidades cariocas e refletimos sobre aspectos do ensino de visualização de dados tanto na graduação quanto no ensino básico. Observamos como a linguagem visual se perde durante o ensino formal. Continuamos com o mesmo tema na semana seguinte.

A sexta edição, em 18/03, intitulou-se 'Educação e Design de Informação' e continuamos a conversar sobre as competências e habilidades que devemos desenvolver com os alunos, por meio do ensino do design de informação. Dezoito pessoas estiveram presentes. Alguns exalunos trouxeram sua experiência, debatendo sobre a valorização pelo mercado de trabalho do profissional qualificado no uso de softwares.

Assim, no evento seguinte - o sétimo, dia 24/03 - convidamos para um papo com alguns alunos e ex-alunos, no intuito de identificarmos os "Primeiros passos na visualização", ou seja, o que seria necessário para começar a fazer visualização de dados. Propusemos uma conversa sobre ferramentas e programação para quem está querendo inserir-se nessa área. Os 13 presentes conversaram sobre a importância da inserção da lógica nos currículos do ensino básico.

O oitavo encontro, em 31/03, trouxe a questão 'Tenho um conjunto de dados, e agora?', instigando a troca de ideias sobre como organizar um banco de dados para possibilitar diversas visualizações. Convidamos um profissional de engenharia de dados para que nos contasse sua experiência nessa área. Aprendemos sobre o funcionamento de softwares de bancos de dados, assim como de processos de extração desses. Terminamos o evento observando a necessidade intrínseca de equipes interdisciplinares.

O nosso nono evento, em 07/04, perguntou-se: 'Pesquisador tem que saber visualização?'.

Discutimos quais ferramentas e estratégias de visualização podem ser utilizadas pelo pesquisador, que busca mostrar seus resultados para um público específico. Analisamos a importância do olhar crítico para o conjunto de dados e a postura ética do pesquisador na construção das visualizações. 
Demos continuidade ao tema na nossa décima edição em 14/04. Convidamos uma especialista em questões relativas à transparência e valores éticos na captação de dados para debater sobre a 'Ética nos dados e visualizações'. Em torno de 40 pessoas, conversamos sobre coleta de dados realizadas pelas redes sociais e o desconhecimento dos usuários sobre esses processos. Este evento também marcou a migração do PapoDado para o Twitter Spaces.

Em 21/04, a décima primeira sessão de encontros tratou da conversa sobre 'Ética, dados e design', no entanto focando em como os dados coletados pelos diversos aplicativos e sites que frequentamos eram utilizados para tornar a experiência do usuário mais proveitosa. Em uma reunião interdisciplinar, formamos um grupo de aproximadamente 16 pessoas.

'Coleta de dados gera navegações personalizadas mais eficientes?' foi o título do décimo segundo encontro em 28/04. No encontro, observamos a utilização de dados por empresas que realizam promoções e ações de marketing e discutimos sua eficácia.

Em 05/05, o tema do décimo terceiro encontro refletimos sobre 'Visualização de dados do setor público', chamando alguns participantes do encontro "DataViz.Rio". A partir de contribuições de profissionais que trabalham no setor público, discutimos projetos que visam a transparência dos dados públicos, assim como pela acessibilidade em visualizações complexas e interativas.

Apresentamos aqui apenas um breve panorama dos temas discutidos até então. A complexidade alcançada nos encontros não poderia ser descrita tão detalhadamente devido à insuficiência do espaço deste artigo. 
Figura 4:mapa dos assuntos abordados durante as 13 sessões do PapoDado já realizadas.

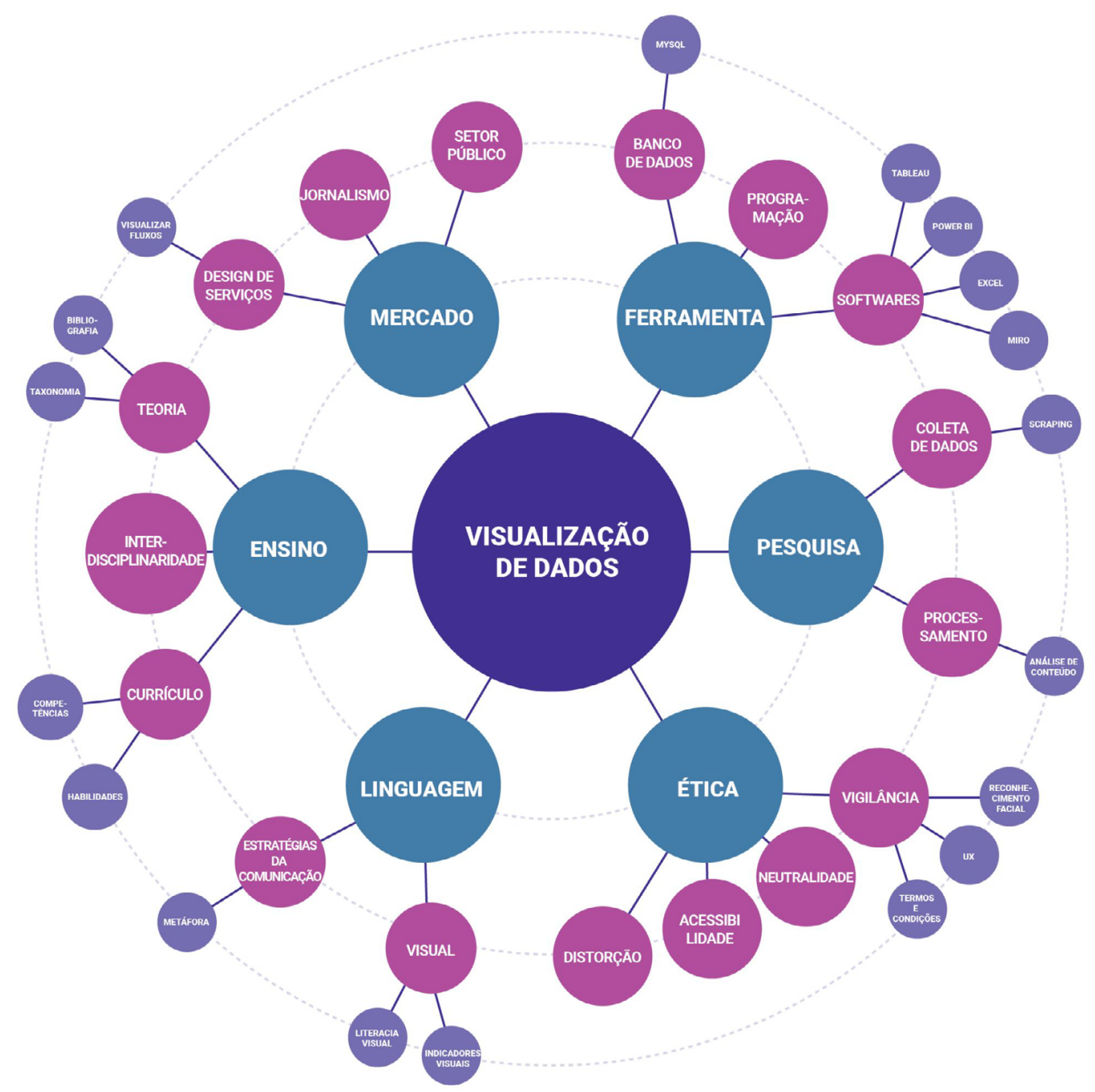

\section{Próximos passos}

Após esses 13 encontros, destacamos, entre muitos aprendizados: a troca relevante de referências, o aprofundamento do campo teórico, o cruzamento de experiências entre profissionais, educadores e pesquisadores, o contato com o mercado de trabalho, a geração de novos vínculos e a aproximação com a comunidade do design de informação e da visualização de dados.

Por estar em ambiente social e pela relativa facilidade de acesso ao espaço de conversa, as plataformas escolhidas permitiram o compartilhamento de experiências horizontalmente, alcançando um perfil de participantes bem diverso. A assistência, de modo geral, foi composta por alunos, professores, pesquisadores e profissionais das mais diversas áreas do conhecimento.

Podemos afirmar que tanta diversidade na realização das sessões favoreceu uma larga navegação pelos assuntos que tangenciam a visualização de dados e o design de informação. De fato, os encontros periódicos têm nos permitido conversas muito atualizadas, refletindo em 
cima de acontecimentos cotidianos e produções contemporâneas, junto a outros interessados no tema.

Os encontros utilizando apenas o áudio, com o objetivo de discutir visualização, provocam a necessidade de descrições verbais aprofundadas, estimulando os participantes a elaborarem discursos que levem a audiência a imaginar a representação gráfica narrada, como num sofisticado exercício de imaginação e interpretação.

As conversas encerram-se nas salas a cada semana, permanecendo compartilhado em nossa memória o conhecimento que vem sendo produzido coletiva e continuamente. No entanto, a impossibilidade de disponibilização do conteúdo de modo assíncrono nas ferramentas utilizadas, vem nos fazendo refletir sobre uma adaptação no formato, no intuito de permitir a gravação dos encontros e seu posterior compartilhamento, ideia ainda em amadurecimento.

\section{Referências}

Kosminsky, D., Castro, B. \& Ludwig, L. (orgs.). (2018). Existência numérica. Rio de Janeiro: Rio Books.

Kirk, A. (2012). Data visualization: a successful design process. Birmingham: Packt Publishing, 2012.

Ramachandran, V (2012). Stanford researchers identify four causes for Zoom fatigue and their simple fixes. California: Stanford News. Disponível em:

<https://news.stanford.edu/2021/02/23/four-causes-zoom-fatigue-solutions>. Acesso em: 10 maio 2021.

Diamond, S. (2011). Visualização de dados: materialidade e mediação. Kosminsky, D., Castro, B., Ludwig, L. (orgs.) (2018). Existência numérica (p. 57). Rio de Janeiro: Rio Books.

Lévy, P. (2003). A inteligência coletiva: Por uma antropologia do ciberespaço (L. P. Rouanet, Trad.; $4^{\circ}$ ed). Edições Loyola.

\section{Sobre os autores}

Luiz Torres Ludwig, MFA, PUC-Rio, Brasil <luiztorresludwig@gmail.com> Joy Helena Worms Till, Dra., PUC-Rio, Brasil <falecom@joytill.com.br> Flavia Nizia da Fonseca Ribeiro, Dra., PUC-Rio, Brasil <flavianizia@gmail.com> 